\title{
Propofol or midazolam for short-term alterations in sedation
}

Owen Boyd MrCP,* C. John Mackay FrCAnaes, $\dagger$ Fiona Rushmer, + E. David Bennett FRCP,* R. Michael Grounds MD FFARCs $\dagger$
It is often necessary to adjust a patient's sedation level while they are in the intensive care unit. The purpose of this study was to compare propofol with midazolam for controlling shortterm alterations in sedation. Twenty-three patients undergoing an interactive procedure, physiotherapy, during mechanical ventilation of the lungs were studied. The patients were randomly assigned to receive infusions of propofol or midazolam for sedation. Sedation was assessed using the method of Ramsay, where 3 is drowsy responding only to commands; and 5 is asleep with a slow response to light glabellar tap. Prior to physiotherapy sedation was deepened from 3 to 5 by increasing the sedative infusion rate, and level 5 was maintained during physiotherapy by adjusting the infusion rate whenever necessary. After physiotherapy, the sedative dose was reduced until level 3 was again achieved. During physiotherapy, sedation level 5 was achieved for $53.9 \%$ of the time with propofol but for only $25.7 \%$ with midazolam $(P<0.01)$. After physiotherapy, those patients sedated with propofol re-awakened to level 3 faster 18.3 $\pm 2.3 \mathrm{~min}$, mean $\pm S E$ ) than those receiving midazolam $(92.8$ $\pm 35.0 \mathrm{~min}, P<0.05$ ). After physiotherapy, a further $1.8 \pm$ 0.5 dose adjustments were required to the midazolam infusion while only $0.4 \pm 0.2$ adjustments were required to the propofol infusion $(P<0.05)$. During physiotherapy $3.0 \pm 0.5$ dose adjustments to the propofol dose were required compared with $3.6 \pm 0.5$ adjustments to the midazolam dose (NS). It is concluded that, during a standardized stimulus, physiotherapy, propofol infusion allowed a desired sedation score to be maintained for more of the time than did infusion of midazolam. Subse-

\section{Key words}

ANAESTHETICS, INTRAVENOUS: midazolam, propofol; HYPNOTICS: midazolam, propofol;

INTENSIVE CARE: sedation.

From the General Intensive Care Unit, Divisions of Medicine* and Anaesthesia $\nmid$ and The Department of Physiotherapy†, St. George's Hospital, London, UK.

Address correspondence to: Dr. Owen Boyd, The General Intensive Care Unit, St. George's Hospital, Blackshaw Rd, London SW17 0QT, UK.

This work has been supported by a grant from ICI

Pharmaceuticals (UK).

Accepted for publication 10th August, 1993. quently, when the infusion rates were reduced, less time was taken to re-awaken to baseline levels after physiotherapy, with fewer adjustments to the infusion rate, in those patients receiving propofol than midazolam.

Aux soins intensifs, il faut fréquemment modifier le niveau de sédation; cette étude compare le propofol au midazolam pour contrôler ces changements de courte durée. Vingt-trois patients sous ventilateur mécanique soumis à des manoeuvres de physiothérapie interactive sont inclus dans létude. Ils reçoivent au hasard en sédation une perfusion de profopol ou de midazolam. La sédation est évaluée selon l'échelle de Ramsey: on accorde 3 pour la somnolence avec réponse aux ordres et 5 au sommeil avec réponse lente à la légère percussion de la glabelle. Avant la physiothérapie, la sédation est approfondie du niveau 3 au niveau 5 en augmentant la perfusion au besoin. Après la physiothérapie, on réduit la perfusion jusqu'au retour au niveau 3. Pendant la physiothérapie, le niveau 5 est atteint pendant $53,9 \%$ de la durée du traitement avec le propofol contre seulement $25,7 \%$ avec le midazolam $(P<0,01)$. Après la physiothérapie, les patients sous propofol reviennent au niveau 3 plus rapidement $(8,3 \pm 2,3$ min, moyenne $\pm S D)$ que ceux qui reçoivent du midazolam $(92,8 \pm 35,0 \mathrm{~min}, P<0,05)$. Après la physiothérapie, le dosage de midazolam nécessite 1,8 \pm 0.5 ajustements comparativement à $0,4 \pm 0,2$ pour le propofol $(P<0,05)$. Pendant la physiothérapie, la posologie du propofol doit être ajustée 3,0 $\pm 0,5$ fois comparativement à 3,6 $\pm 0,5$ pour celle du midazolam (NS). En conclusion, pendant une stimulation standardisée, la perfusion de propofol permet de maintenir le niveau de sédation désiré plus souvent que la perfusion de midazolam. Par la suite, lorsqu'on diminue la perfusion, le temps requis pour le retour à la ligne de base nécessite moins d'ajustements pour le propofol que pour le midazolam.

Most patients admitted to intensive care units, who are subsequently ventilated artificially, require some form of sedation during at least part of their stay and it is common practice to use either midazolam or propofol by continuous intravenous infusion. It has been shown ${ }^{1-6}$ that over a 24-hr period both midazolam and propofol offer good quality of sedation and that the quicker wake up time that is seen with propofol may mean that this agent has benefits over midazolam. These studies, however, 
have not considered the ability to control sedation accurately when short-term changes are needed, and a potential advantage of one agent over the other in this area may be missed by studies considering a longer time frame. Short-term alterations in sedation are often required during procedures such as placing intravenous catheters, changing of position, hospital transfers or physiotherapy. This study was designed as a prospective, randomized, double-blind comparison of propofol with midazolam for maintaining preset sedation levels in postoperative patients undergoing a standard physiotherapy regimen during mechanical ventilation of the lungs.

\section{Methods \\ Informed consent for participation in the study was ob- tained from 23 patients prior to elective repair of ab- dominal aortic aneurysm. The study had been approved by our institutional ethics committee and after enrollment patients were randomly assigned to a group receiving pro- pofol by continuous infusion or a group receiving mi- dazolam by continuous intravenous infusion for post- operative sedation. Quality of sedation was then assessed and compared between each group of patients during a standard physiotherapy regimen.}

\section{Operative anaesthetic management}

All patients were pre-medicated with $50 \mathrm{mg}$ meperidine and $25 \mathrm{mg}$ promethazine hydrochloride one hour before surgery. Induction of anaesthesia was with propofol (100 $\mathrm{mg}$ to $200 \mathrm{mg}$, to induce sleep) and fentanyl $(100 \mu \mathrm{g})$ iv. Maintenance of anaesthesia was by continuous propofol infusion with intermittent $100 \mu \mathrm{g}$ bolus doses of fentanyl administered incrementally until a total dose of $25 \mu \mathrm{g} \cdot \mathrm{kg}^{-1}$ had been given. Propofol was infused using an Ohmeda 9000 infusion pump ${ }^{7}$ to achieve a target concentration of 3 to $6 \mu \mathrm{g} \cdot \mathrm{ml}^{-1}$ judged adequate by the control of blood pressure and heart rate, and the absence of sweating. The rate of infusion was controlled using a Psion II computer which varied the rate of infusion to allow predicted steady state plasma concentrations of propofol. The computer prediction of plasma concentration of propofol is based on a three compartment kinetic model for the drug taking into account the patient's age and weight; the accuracy of the prediction has previously been described. ${ }^{8}$ Muscle relaxation was achieved using vecuronium $0.1 \mathrm{mg} \cdot \mathrm{kg}^{-1}$ as an initial dose and incremental doses of $0.05 \mathrm{mg} \cdot \mathrm{kg}^{-1}$ when required as determined by use of a peripheral nerve stimulator. Artificial ventilation (intermittent positive pressure ventilation) was with oxygen enriched air with $\mathrm{FIO}_{2}$ 0.4. A lumbar epidural catheter was inserted using standard aseptic technique prior to the operation for postoperative pain relief.
At the end of the operation the patients were transferred to the intensive care unit (ICU) where their lungs were electively artificially ventilated in the routine fashion in our unit. Patients were randomly allocated to receive either propofol or midazolam by continuous infusion for sedation during mechanical ventilation in the ICU. Analgesia was given by continuous epidural infusion of fentanyl $100 \mu \mathrm{g}$ in $40 \mathrm{ml} 0.25 \%$ bupivacaine. The infusion rate was adjusted in the range $0-8 \mathrm{ml} \cdot \mathrm{hr}^{-1}$ by the ICU nursing staff as required by the patient to relieve pain.

\section{Assessments of sedation level}

Throughout the study, sedation level was judged by using the bedside scale of Ramsay et al: ${ }^{9}$ (1) anxious and agitated; (2) cooperative orientated and tranquil; (3) drowsy responds to commands only; (4) asleep with a brisk response to light glabellar tap; (5) asleep with a slow response to light glabellar tap; (6) asleep with no response to light glabellar tap. In the immediate postoperative period the sedation score was judged by the nursing staff, who were free to titrate the sedative agent as appropriate to obtain a score of 3 . This is normal practice on our ICU and the nursing staff are skilled at achieving a desired sedation level. For the hour prior to physiotherapy, during the physiotherapy and until the patient had reawoken to sedation score 3 after physiotherapy one of the investigators (CJM) assessed sedation score. One investigator was chosen to avoid inter-observer bias and he was blinded to the sedative agent being infused by holding the syringes containing the drug and the infusion control apparatus behind opaque screening. The infusion lines were hidden in opaque foil as far as the patients' skin, in the same way that sodium nitroprusside is screened from light.

\section{Postoperative protocol}

At the end of 24-hr ventilation all the patients underwent a standard physiotherapy regimen (see below). Before commencing physiotherapy it was confirmed that the patients were free from pain and then the infusion rate of their sedative agent was increased from the baseline rate that gave a sedation score of 3 to a rate titrated to provide a sedation score of 5 . Once this had been done physiotherapy was started. During physiotherapy, assessments of the patients' sedation level were made every two minutes. The infusion rate of the sedative agent was adjusted to maintain a sedation score of 5 by increasing the infusion rate every two minutes if a score of $<5$ was found. If an increase was necessary the sedative agent was increased by $25 \%$ each time. At the end of physiotherapy the rate of infusion of the sedative agent was reduced to that rate being used prior to the study to allow the patient to re-awaken to sedation level 3 . The time taken 
TABLE I Details of the patients, no differences are significant

\begin{tabular}{lll}
\hline & Propofol & Midazolam \\
\hline$n$ & 10 & 9 \\
Age, yr & $67.2 \pm 2.3$ & $70.9 \pm 3.6$ \\
Apache Il score & $10.9 \pm 0.8$ & $12.8 \pm 1.2$ \\
Male:female & $9: 1$ & $8: 1$ \\
\hline
\end{tabular}

to reawaken to a level of 3 was recorded. Assessments of the patients' sedation scores were continued every two minutes for $20 \mathrm{~min}$ and then every five minutes for a further $30 \mathrm{~min}$. During this time, the rate of infusion of the sedative agent was reduced by a further $50 \%$ every ten minutes if the patient's sedation score was greater than 3 , the number of these infusion rate adjustments was recorded. If patients had not returned to sedation score 3 within $50 \mathrm{~min}$, the time to reach a score of 3 after that time was recorded.

At the same times as assessments of the patient's sedation score was performed, heart rate from ECG recording, arterial blood pressure from intra-arterial catheter and infusion rate of the sedative agent were recorded. The dose of epidural infusion providing adequate analgesia immediately prior to starting physiotherapy was also recorded.

\section{Physiotherapy regimen}

Each patient was assessed by the physiotherapist prior to treatment for suitability of treatment and analysis of any respiratory problems. Physiotherapy was carried out for 12-16 min with the patient lying on alternate sides with one pillow under the head. This position was standardized for the purposes of the trial. The right chest and the left chest were treated in turn. All patients received manual hyperinflation with $100 \%$ oxygen using a $2 \mathrm{~L}$ re-breathing bag and a flow rate $>10 \mathrm{~L} \cdot \mathrm{min}^{-1}$. The technique used was four slow deep breaths with quick release followed by four small breaths at tidal volume. Shaking on expiration was used if secretions were audible and $0.9 \%$ saline was instilled if secretions were tenacious. This was followed by suction with an airflow catheter using a negative pressure of $15-20 \mathrm{kPa}$. This technique was repeated on both sides. At the end of the physiotherapy the patient was reattached to the ventilator and turned supine.

\section{Statistical analysis}

Two-way analyses of variance for group and time were used to compare heart rate and mean arterial pressure, both for absolute values and changes from baseline. Fisher's exact test was used to compare absolute data, including the percentage of time that the desired sedation score was or was not achieved during physiotherapy,

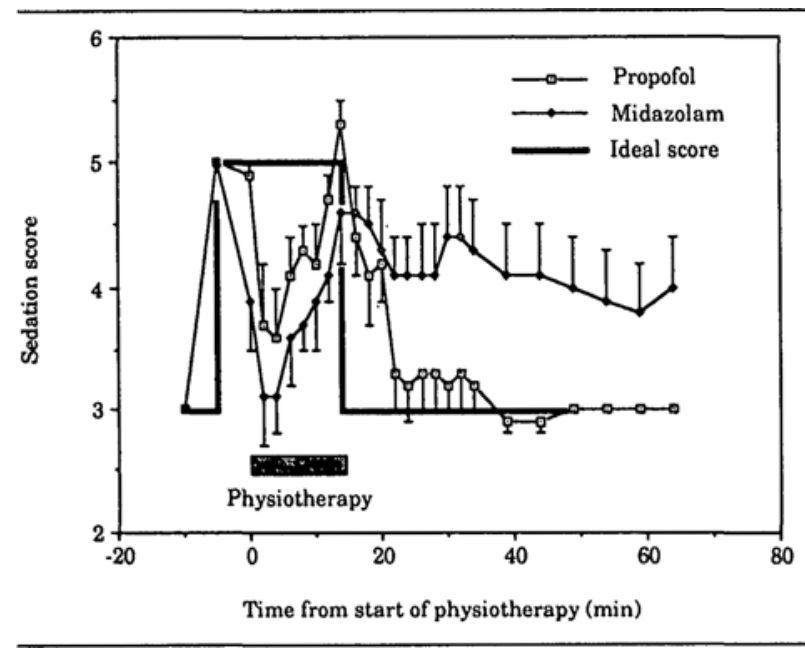

FIGURE 1 Sedation score before, during and after physiotherapy (mean $\pm \mathrm{SE}$ ), with the ideal sedation score shown.

which was generated as a summary statistic to avoid the problems of multiple comparisons. ${ }^{10}$ The Whitney-Mann $\mathrm{U}$ test was used to compare other data. All results are mean $\pm \mathrm{SE}$ and $P<0.05$ is taken as significant.

\section{Results}

Twenty-three patients were initially enrolled in the study but four patients, two randomised to receive propofol and two to receive midazolam, were subsequently withdrawn, as three had a second operation due to postoperative bleeding within the first $24 \mathrm{hr}$ and one died. The results presented are from analysis of the remaining 19 patients.

\section{Demographic details}

There were no differences between the two groups with regard to age or Apache II score (Table I). All patients had abdominal aortic aneurysm repair.

\section{Quality of sedation}

Figure 1 shows the pattern of changes in sedation score during and after physiotherapy. All patients were maintained without difficulty at sedation level 3 prior to deepening the level of sedation before physiotherapy. There was no difference in the time taken to deepen the patients to a sedation score of 5 prior to physiotherapy (Table II). During physiotherapy and after physiotherapy patients in the propofol group achieved the target sedation score $53.9 \%$ of the time, significantly more often than patients in the midazolam group who only achieved the target sedation score for $25.7 \%$ of the time. After physiotherapy re-wakening to sedation level 3 was more rapid in the propofol than in the midazolam group.

\section{Haemodynamic results}

There were no differences in mean arterial pressure and 
TABLE II Quality of sedation

\begin{tabular}{lll}
\hline & Propofol & Midazolam \\
\hline $\begin{array}{l}\text { Time to sedate to level 5 } \\
\text { (median) (min) }\end{array}$ & $18.1 \pm 3.9(15)$ & $9.9 \pm 1.2(10)$ \\
$\begin{array}{l}\text { Time to re-awaken to level 3 } \\
\quad \text { min) }\end{array}$ & $8.3 \pm 2.3^{*}$ & $92.8 \pm 35.0$ \\
$\begin{array}{c}\text { Time at target sedation level } \\
\text { during physiotherapy }\end{array}$ & $53.9 \% \dagger$ & $25.7 \%$ \\
\hline
\end{tabular}

$* P<0.05$ (Whitney-Mann U test).

$\dagger P<0.00$ l (Fisher's exact test).

heart rate between the two groups during the study period (Figure 2).

\section{Drug infusion requirements}

There were fewer dose adjustments required to the propofol infusion after the period of physiotherapy than were required to the midazolam infusion rate $(P<0.05$, Table III). During physiotherapy, there were no differences in the number of times that the rate of infusion of the sedative drug was changed (Table III and Figure 3). There was no difference between the two groups in the rate of epidural infusion prior to commencing physiotherapy and all patients were free from pain before, during and after receiving physiotherapy for the study protocol.

\section{Discussion}

This study compared the effectiveness of sedative infusions of propofol and midazolam in controlling shortterm alterations in sedation level in postoperative patients undergoing physiotherapy during mechanical ventilation of the lungs. The results showed that during physiotherapy, propofol infusion allowed a desired sedation score to be maintained for a greater percentage of the time, than with midazolam, although there was no difference in the number of times the propofol infusion rate or the midazolam infusion rate had to be adjusted during the physiotherapy. After physiotherapy, patients who had received propofol as the sedative agent re-awoke to baseline sedative level more quickly than the patients who had received midazolam and required fewer adjustments in the sedative infusion rate. There were no differences in heart rate or blood pressure seen with the two agents.

There are a number of studies which have compared the use of propofol and midazolam for ICU sedation, usually in the postoperative period. ${ }^{1-6}$ However, these have been concerned with a broad overview of sedation and we are not aware of any studies that have considered the ease with which the different agents can control shortterm changes in sedation. Medical and nursing staff perceive ease of titration of one sedative agent compared to another as a potential major advantage ${ }^{1}$ and this study
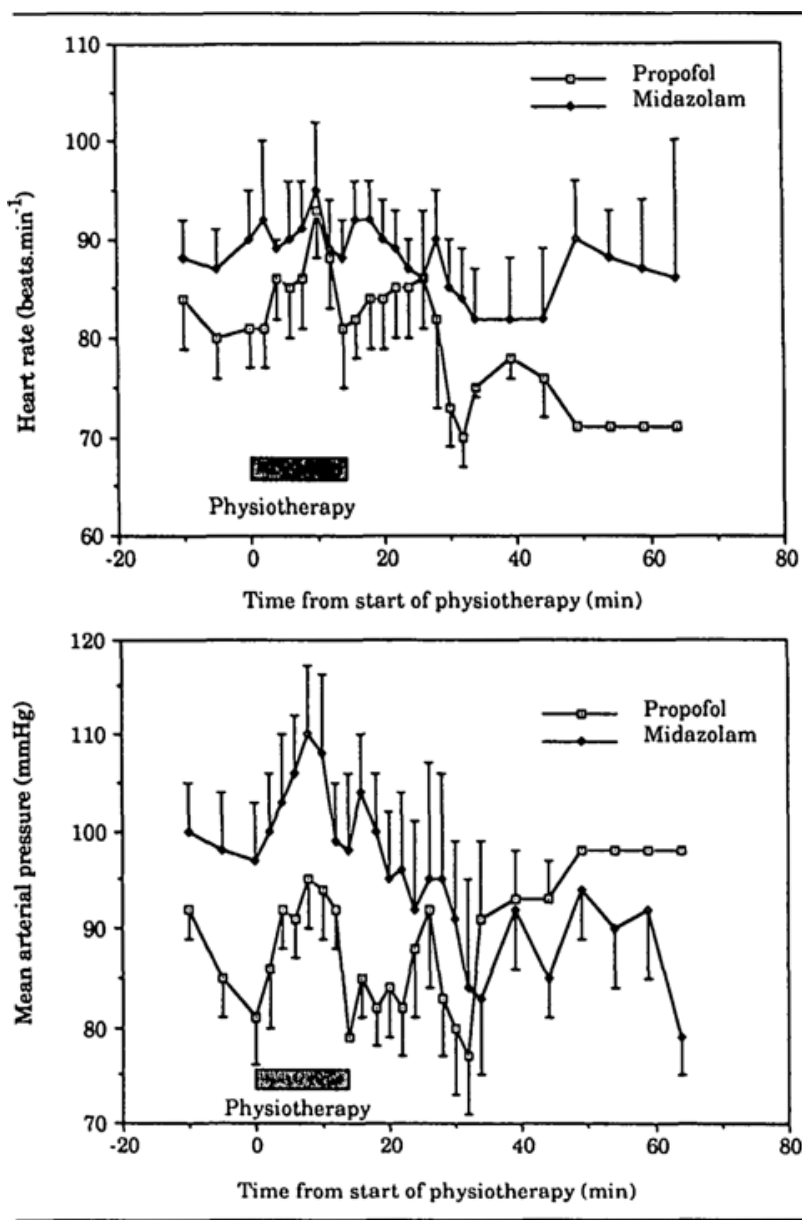

FIGURE 2 Heart rate and mean arterial pressure before, during and after physiotherapy (mean $\pm \mathrm{SE}$ ).

was therefore designed to compare, in as relevant a way as possible, the ability of propofol and midazolam to control for short-term changes in sedation. We had previously noticed that during interactive procedures ICU staff tended to deepen sedation by increase in infusion rate of the given agent rather than by a more complicated, alternative regimen involving administration of a bolus of sedative followed by infusion at an increased rate. We therefore designed this study to reflect, as far as possible, current practice in our ICU by altering sedation infusion rates to change sedation level. Other techniques for altering blood concentrations of drugs such as bolus administration of propofol or midazolam at the start of physiotherapy followed by increased rate of sedation infusion might have led to more similar results for the percentage of time that the target sedation level was maintained during physiotherapy with each agent. However, the amount of propofol or midazolam given by bolus injection to achieve a desired sedation level is not known and is likely to depend on numerous factors, and it is probable that the new infusion rate would also have to 
TABLE III Drug infusions

\begin{tabular}{lll}
\hline & Propofol & Midazolam \\
\hline $\begin{array}{l}\text { Baseline dose (range), } \mathrm{mg} \cdot \mathrm{hr}^{-1} \\
\begin{array}{l}\text { Bolus of sedative given during physiotherapy } \\
\text { (range), } \mathrm{mg}\end{array}\end{array}$ & $133 \pm 17.3(50-200)$ & $4.02 \pm 1.41(0.2-13.5)$ \\
$\begin{array}{l}\text { Number of infusion adjustments during } \\
\text { physiotherapy }\end{array}$ & $235 \pm 17.3(150-320)$ & $7.83 \pm 2.13(1-17.5)$ \\
$\begin{array}{l}\text { Number of infusion adjustments after } \\
\text { physiotherapy and after the initial reduction }\end{array}$ & $3.0 \pm 0.5$ & $3.6 \pm 0.5$ \\
$\quad$ to baseline rate & $0.4 \pm 0.2^{*}$ & $1.8 \pm 0.5$ \\
Epidural infusion rate, $\mathrm{ml} \cdot \mathrm{hr}^{-1} \dagger$ & $5.6 \pm 0.6$ & $5.3 \pm 1.1$ \\
\hline
\end{tabular}

$* P<0.05$ (Whitney-Mann U test).

† Epidural fentanyl $100 \mu \mathrm{g}$ in $40 \mathrm{ml} 0.25 \%$ bupivacaine.

be altered as physiotherapy progressed. We would also expect that the difference in the re-awakening time would have been greater if a bolus protocol had been used and that there might have been greater haemodynamic instability than the very minor changes seen in our study.

Although this study was concerned with the control of sedation in patients undergoing physiotherapy during mechanical ventilation of the lungs, the results may have wider implications. Many authorities maintain artificially ventilated patients in their intensive care units, at a level of sedation where it is possible to communicate with the patient but where anxiety is relieved and where it is pos-

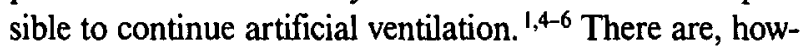
ever, many occasions in an intensive care unit when it is required to alter a patient's level of sedation. When synchronising a patient to artificial ventilation, carrying out invasive procedures, moving the patient and carrying out procedures such as wound dressing or cleaning, as well as when performing physiotherapy, the patient is more easily managed and distress will be relieved if the level of sedation is temporarily deepened. When these procedures are being performed the patient's level of sedation is usually deepened to a Ramsay score of 5 (asleep with a slow response to light glabellar tap). During physiotherapy for example, it is desirable to have the patient at this deeper level of sedation to relieve distress, to keep airway pressures lower during manual lung inflations and to prevent biting on the endotracheal tube.

Physiotherapy was chosen as a model for an interactive procedure in this study because it was possible to standardise the protocol, all patients would be required to undergo physiotherapy and because physiotherapy provides a considerable stimulus to the patient. We also attempted to minimise the potential influence of other factors on our patients by providing continuous epidural analgesia at a constant rate sufficient to relieve pain, and saw no differences in the rate of administration of epidural analgesia in the two groups. We studied patients having just one type of operation to minimise potential
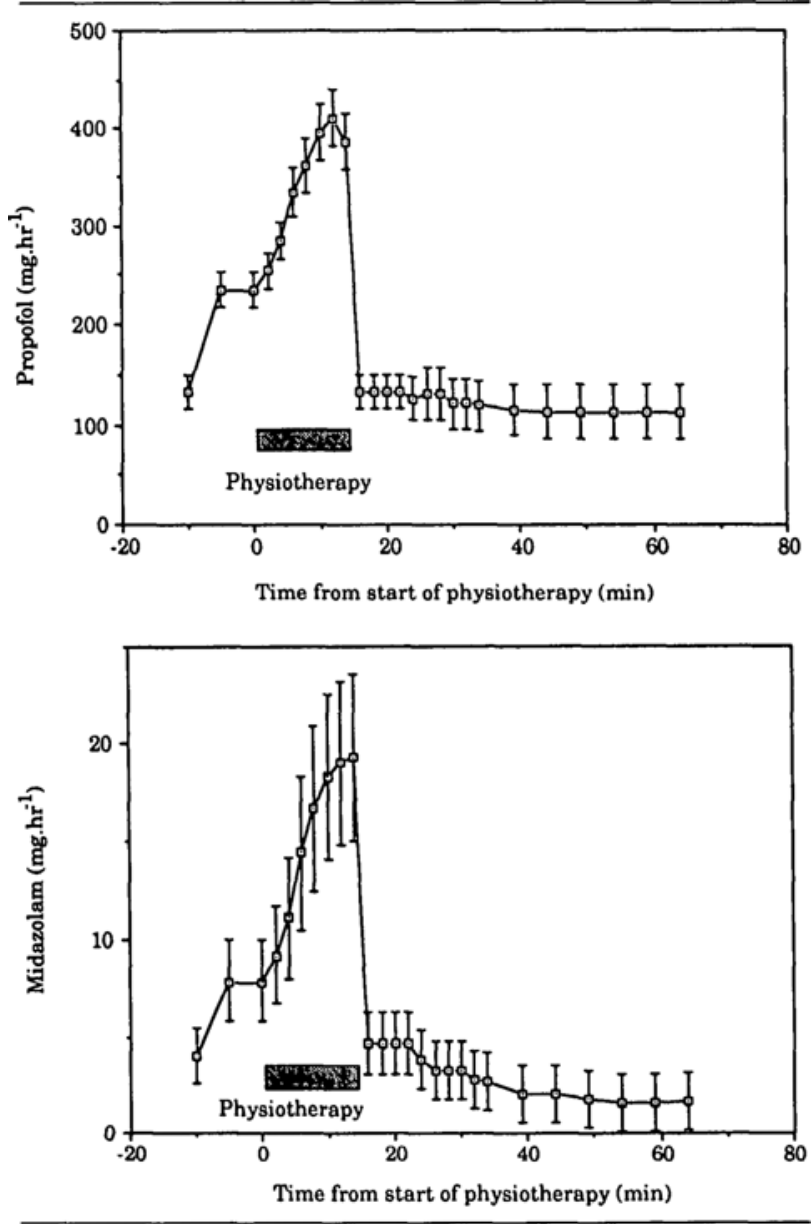

FIGURE 3 Infusion rates of propofol and midazolam before, during and after physiotherapy (mean $\pm \mathrm{SE}$ ).

variations, and used a standardised short-acting intravenous anaesthetic technique for general anaesthesia. The short half-life of propofol makes it unlikely that the intraoperative infusion would have any important effect on sedation $24 \mathrm{hr}$ later, and the fact that both patient groups received the same anaesthetic should exclude any effect 
of this on subsequent comparisons of sedation. Also, we have recently shown that propofol anaesthesia leads to improved cardiac function compared with isoflurane in patients undergoing peripheral vascular surgery and this has become our usual practice for all patients having vascular surgery."

Although most studies have shown little difference between propofol or midazolam as far as cardiovascular changes are concerned, ${ }^{3,5,6}$ one study has suggested that there may be a greater hypotensive effect associated with the use of propofol than with midazolam. ${ }^{12}$ The lack of difference in these cardiovascular variables between the two groups in our study confirms the majority of earlier studies showing no difference between propofol and midazolam. The similar cardiovascular results may have been due to the patients' stable haemodynamic states with adequate cardiac pre-loading with fluid in the postoperative phase, and may also have been influenced by the epidural analgesia used.

To some extent, the findings of this study could have been anticipated by differences in the pharmacokinetic profiles of propofol and midazolam. Propofol has a much shorter $t_{1 / 2}$ than midazolam with awakening within ten minutes even after a prolonged infusion, ${ }^{13,14}$ while the $t_{1 / 2}$ of midazolam ranges from one to four hours and can be unpredictable in critically ill patients. ${ }^{15}$ The much shorter $t_{1 / 2}$ of propofol might have led to an increase in the number of dose adjustments during the physiotherapy regimen than with midazolam and a reduced quality of sedation with over-sedation and under-sedation from the required level during the interactive procedure. This study was designed to compare only the short-term titratability of propofol and midazolam infusions during postoperative sedation and has been able to answer these points. Titratability is only one of many factors that can improve patient care, albeit an important practical consideration; further investigations of differences in patient satisfaction, duration of ICU stay, time to extubation and other potential differences need to be undertaken to build up a better picture of the benefits of different agents for different patient groups.

In conclusion we have shown in a randomized trial that propofol provides better control of short-term alterations in sedation than midazolam. This is important because short-term alterations of sedation are frequently required in patients in intensive care units to facilitate a number of procedures, and our results may indicate an important advantage to the use of propofol for ICU sedation compared with midazolam.

\section{Acknowledgements}

We would like to thank the medical, nursing and physiotherapy staff for their help with this study, and the Vascular Unit for permission to study their patients.

\section{References}

1 Grounds RM, Lalor JM, Lumley J, Royston D, Morgan $M$. Propofol infusion for sedation in the intensive care unit: preliminary report. B M J 1987; 294: 397-400.

2 Newman LH, McDonald JC, Walace PGM, Ledingham $I M$. Propofol infusion for sedation in intensive care. Anaesthesia 1987; 42: 929-37.

3 Aitkenhead AR, Peppermen ML, Willatts SM, et al. Comparison of propofol and midazolam for sedation in critically ill patients. Lancet 1989; 2: 704-9.

4 Harris CE, Grounds RM, Murray AM, Lumley J, Royston $D$, Morgan $M$. Propofol for long-term sedation in the intensive care unit. A comparison with papaveretum and midazolam. Anaesthesia 1990; 45: 366-72.

5 Snellen F, Lauwers P, Demeyere R, Byttebier G, Van Aken $H$. The use of midazolam versus propofol for short term sedation following coronary artery bypass grafting. Intensive Care Med 1990; 16: 312-6.

6 McMurray TJ, Collier PS, Carson IW, Lyons SM, Ellion P. Propofol sedation after open heart surgery. A clinical and pharmacokinetic study. Anaesthesia 1990; 45: 322-6.

7 Stokes DN, Peacock JE, Lewis R, Hutton P. The Ohmeda 9000 syringe pump. The first of a new generation of syringe drivers. Anaesthesia 1990; 45: 1062-6.

8 White M, Kenny GNC. Intravenous propofol anaesthesia using a computerised infusion system. Anaesthesia 1990; 45: 204-9.

9 Ramsay MAE, Savege TM, Simpson BRJ, Goodwin R. Controlled sedation with alphaxalone-alphadolone. B M J 1974; 2: 656-9.

10 Matthews JNS, Altman DG, Campbell MJ, Royston P. Analysis of serial measurements in medical research. B M J 1990; 300: 230-5.

11 Boyd O, Murdoch LJ, Mackay CJ, Bennett ED, Grounds $R M$. The cardiovascular changes associated with equipotent anaesthesia with either propofol or isoflurane: particular emphasis on right ventricular function. Acta Anaesthesiol Scand (in press).

12 Chaudhri S, Kenny GNC. Sedation after cardiac bypass surgery: comparison of propofol and midazolam in the presence of a computerized closed loop arterial pressure controller. Br J Anaesth 1992; 68: 98-9.

13 Beller JP, Pottecher T, Lugnier A, Mangin P, Otteni JC. Prolonged sedation with propofol in ICU patients: recovery and blood concentration changes during periodic interruptions in infusion. Br J Anaesth 1988; 61: 583-8.

14 Cockshott ID. The pharmacokinetics of propofol in the ICU patient. Journal of Drug Development 1991; 4 (Suppl 3): 29-36.

15 Dirksen MSC, Vree TB, Driessen JI. Clinical pharmacokinetics of long-term infusion of midazolam in critically ill patients - preliminary results. Anaesth Intensive Care 1987; 15: 440-4. 